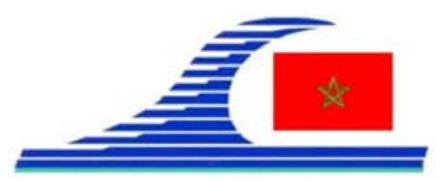

Conférence Méditerranéenne Côtière et Maritime EDITION 2, TANGER, MAROC (2011)

Coastal and Maritime Mediterranean Conference

Disponible en ligne - http://www.paralia.fr - Available online

\title{
Modélisation tridimensionnelle de l'influence de la bathymétrie et des courants sur la dispersion des polluants dans la baie de Tanger (Maroc)
}

\author{
Aicha BELCAID ${ }^{1,2}$, Georges LEPALEC ${ }^{2}$, \\ Abdeslam DRAOUI ${ }^{1}$, Philippe BOURNOT ${ }^{2}$
}

1 Laboratoire d'Energétique - Equipe de recherche en Transferts Thermiques et Energétique (ETTE), Université Abdelmalek Essaâdi, Faculté des Sciences et Techniques de Tanger, Maroc. belcaid_aicha@yahoo.fr; a.draoui@fstt.ac.ma

2 IUSTI, UMR 6595, Technopôle de Château-Gombert, 5 rue Enrico Fermi, 13453 Marseille Cedex 13, France, Marseille, France. georges.lepalec@univmed.fr ; philippe.bournot@univmed.fr

\section{Résumé :}

L'étude est basée sur la simulation numérique du processus de la pollution côtière par les eaux usées rejetées à travers des oueds dans la baie de Tanger- Maroc. Un processus régi par les courants engendrés par la marée, le vent et les variations importantes de débit des différents oueds qui s’y déversent. Étudier expérimentalement un tel problème est couteux et parfois impossible, d'où l'idée d'une étude numérique pour comprendre les différents phénomènes qui régissent la dispersion de polluants dans la baie et prédire le niveau de la dégradation de la qualité de ses eaux. La simulation a été faite à l'aide du code de calcul Fluent, dans le but d'étudier l'influence de la bathymétrie, des courants de la marée et du vent sur la dispersion de polluants dans la baie. Les constatations de la modélisation en bidimensionnelle ont été utilisées pour passer ensuite à une modélisation en tridimensionnelle (3D) avec une profondeur de $15 \mathrm{~m}$ tout en respectant la vraie bathymétrie de la baie afin de mieux s'approcher de la réalité et de cerner physiquement ce processus de la dispersion sous réserve de certaines hypothèses.

Mots-clés :

Pollution côtière - Dispersion - Marée - Vent - Turbulence - Modélisation - Fluent

\section{Introduction}

Dans la plupart des zones urbaines, la majeure partie des eaux usées sont évacuées sans traitement dans des voies d'eau naturelles, ce qui entraîne naturellement une pollution organique. Les conséquences de cette pollution sont catastrophiques pour presque tous les organismes constituant les peuplements des milieux néritiques. Si la zone de rejet qui est d'une pollution purement organique- et son panache ne sont pas contrôlés de manière appropriée, les effluents peuvent revenir le long des côtes sans avoir été suffisamment dilués et risquent de contaminer les zones de pêche, d'élevage ou de 
La connaissance de la Mer:

un vecteur du développement durable en Méditerranée

ramassage de coques, ..., ainsi que les plages (PROJET SMAP III, 2007). Or toute la partie de la côte marocaine, et notamment la baie de Tanger, est bordée de plages très fréquentées par les touristes, ainsi que de zones de pêche. Des analyses sédimentologiques des eaux de surface de cette baie ont montré une contamination saisonnière des côtes, dont l'évolution spatiale et temporelle s'aggrave par l'intervention de nombreux phénomènes présents.

Cette étude traite physiquement et numériquement le modèle hydrodynamique de la diffusion de polluants due aux eaux usées de la baie de la ville de Tanger, à l'aide du logiciel de calcul CFD FLUENT (FLUENT INC., 2005). Nous avons répertorié les différents paramètres physiques nécessaires à la modélisation, puis effectué le suivi numérique de cette pollution pour un cycle de 24 heures d'une marée semi-diurne et pendant deux périodes de l'année; hivernale et estivale. Pour se faire on a résolu numériquement les équations moyennes de Navier-Stokes écrites en moyenne de Reynolds et couplées aux équations de la conservation de l'énergie et de la concentration.

\section{Equations et Conditions aux limites}

\subsection{Equations du problème}

Les équations qui régissent le problème traité, sont celles des écoulements turbulents et incompressibles écrites en décomposition de Reynolds pour la vitesse, la température et la concentration, en une composante moyenne et un terme de fluctuation :

$\bar{u}=u+u^{\prime} \quad ; \quad \bar{T}=T+T^{\prime} \quad ; \quad \bar{C}=C+C^{\prime}$

Et exprimant respectivement la conservation de la masse, de la quantité de mouvement, de l'énergie et de concentration :

$$
\begin{aligned}
& \frac{\partial \overline{u_{i}}}{\partial x_{i}}=0 \quad i=1,2,3 \\
& \frac{\partial \overline{u_{i}}}{\partial t}+\overline{u_{j}} \frac{\partial \overline{u_{i}}}{\partial x_{j}}=-\frac{1}{\rho} \frac{\partial \bar{P}}{\partial x_{i}}+\frac{\partial}{\partial x_{j}}\left[v\left(\frac{\partial \overline{u_{i}}}{\partial x_{j}}+\frac{\partial \overline{u_{j}}}{\partial x_{i}}\right)-\overline{u_{i}^{\prime} u_{j}^{\prime}}\right]+\bar{f}_{i} \\
& \frac{\partial \bar{T}}{\partial t}+\overline{u_{i}} \frac{\partial \bar{T}}{\partial x_{i}}=\frac{\partial}{\partial x_{i}}\left(a \frac{\partial \bar{T}}{\partial x_{i}}-\overline{u_{i} T^{\prime}}\right)
\end{aligned}
$$

Dans cette étude, nous considérons le polluant: l'eau non salée (l'eau douce) issue des embouchures des oueds.

$$
\rho \frac{\partial \bar{C}}{\partial t}+\rho \frac{\partial\left(u_{i} \bar{C}\right)}{\partial x_{i}}=D \frac{\partial}{\partial x_{i}}\left(\frac{\partial \bar{C}}{\partial x_{i}}\right)
$$

Avec $C$ la salinité et $D$ le coefficient de diffusion du sel, considéré constant.

Le modèle de fermeture choisi pour ce système est le modèle $k-\varepsilon$, qui est largement utilisé (COUSTEIX, 1989 ; DUMAS et al., 1976). 


\subsection{Conditions aux limites}

- Du côté de l'océan atlantique, on a imposé une vitesse constante des courants de la marée et le flux arrive perpendiculairement à la frontière ouverte.

- Pour les cinq oueds qui versent dans la baie et la canalisation majeure de la ville de Tanger, on a représenté leurs débits moyens (en hiver et en été) en imposant une vitesse d'écoulement constante et normale aux différentes embouchures. La température correspondante est prise constante au cours du temps avec une valeur moyenne de $294^{\circ} \mathrm{K}$ pendant la période hivernale.

- Pour le nord (côté espagnol) et l'est (côté méditerranée), on les a considérés des sorties avec une condition qui correspond à la continuité d'écoulement des eaux.

- Sur les côtes, nous avons imposé une vitesse d'écoulement nulle en supposant qu’il s’agit d'une paroi imperméable.

Finalement, à l'instant initial $(t=0)$, la densité $\left(1030 \mathrm{~kg} / \mathrm{m}^{3}\right)$, la température $\left(291^{\circ} \mathrm{K}\right)$ et la salinité (36 g/l) de l'eau salée ont été imposées à l'ensemble de la baie.

\section{Résultats et discussions}

La figure 1 représente pendant la période hivernale, la dispersion de polluants depuis les embouchures des oueds, étudiée sur le modèle de la baie dans sa vraie bathymétrie. Cette dispersion est présentée par les isovaleurs de la fraction massique en polluant pour un cycle de 24 heures (soit 2 cycles d'une marée semi-diurne caractérisée par l'alternance entre une marée montante et une marée descendante au bout de chaque 6 heures).

D'après les résultats, les rejets des oueds ont tendance à se rencontrer au cours du temps. Sous l'effet des courants de la marée montante, la propagation de polluants vers l'ensemble de la baie se trouve relativement freinée. A l'arrivée de la marée descendante, la propagation des rejets se rafraichit en étendant la zone de pollution sur la totalité de la baie et surtout le long de la côte. Le processus de la propagation reprend la même allure au retour des deux marées et le régime d'écoulement se stabilise vers la fin des 24 heures. Notons que la modélisation 3D donne des résultats sensiblement différents par rapport au 2D (BELCAID et al., 2011), mettant en évidence une pollution plus importante. Cette différence était le résultat d'un calcul plus précis en 3D avec un maillage trop raffiné de l'ordre de 3 millions cellules contre 200000 cellules en 2D.

\section{Conclusions}

Nos résultats s'avèrent bien conformes aux constatations d'un certain nombre d'études physico-chimiques et sédimentologiques qui révèlent que la pollution est due essentiellement aux rejets des oueds. Les zones affectées étant identiques à celles de notre étude (EL HATIMI et al., 2002 ; Amendis, Rapports de l'étude de la faisabilité de l'émissaire en mer de la zone de Tanger, 2002). Ceci confirme la prépondérance des mécanismes de flux et de reflux des écoulements dans ce processus de propagation de la 
La connaissance de la Mer :

un vecteur du développement durable en Méditerranée

pollution, et c'est la raison pour laquelle il convient de modéliser ces mécanismes le plus précisément possible.

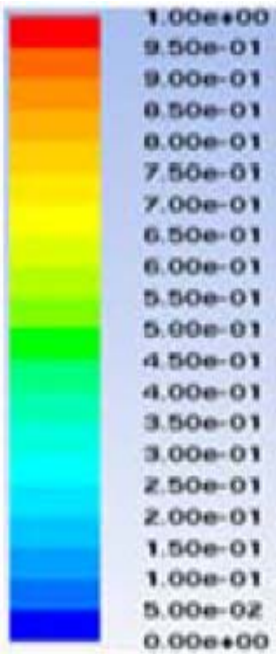

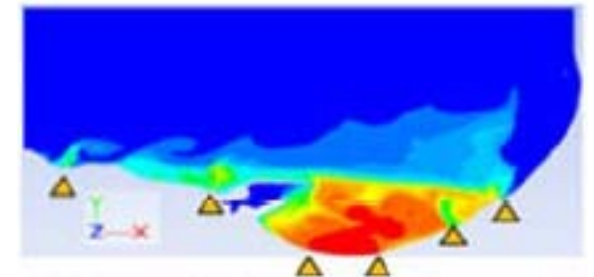

(a) marée montante après 6 heures

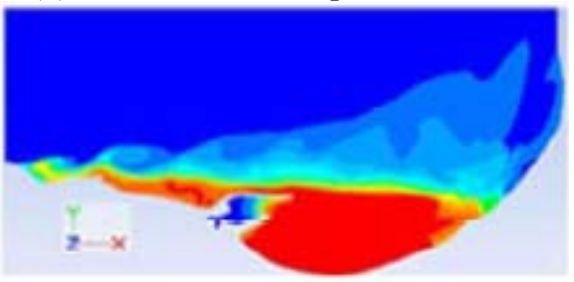

(c) marée montante après 18 heures

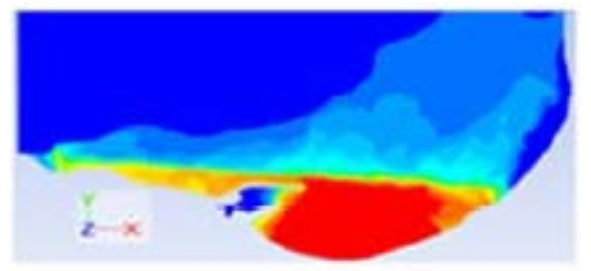

(b) marée descendante après 12 heures

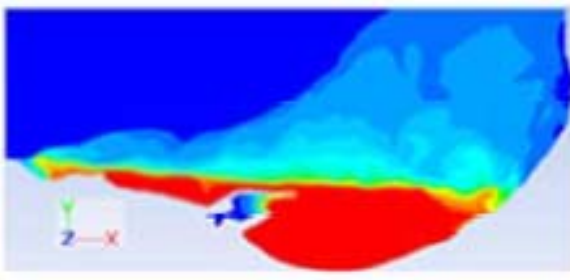

(d) marée descendante après 24 heures

Figure 1. Fraction massique en polluants à la surface libre de la baie.

\section{Nomenclature :}

$a:$ Diffusivité thermique du fluide $\left(\mathrm{m}^{2} \cdot \mathrm{s}^{-1}\right)$

$D$ : Coefficient de diffusion $\left(\mathrm{m}^{2} \mathrm{~s}^{-1}\right)$

$C$ : Concentration de l'espèce $\left(\mathrm{g} \mathrm{L}^{-1}\right)$

$T$ : Température $\left({ }^{\circ} \mathrm{K}\right)$

$p:$ Pression statique $(\mathrm{Pa})$

$t:$ Temps (s)

$u_{i}$ : Composante de vitesse $\left(\mathrm{m} \mathrm{s}^{-1}\right)$

$u_{i}^{\prime}$ : Fluctuation de la composante de vitesse

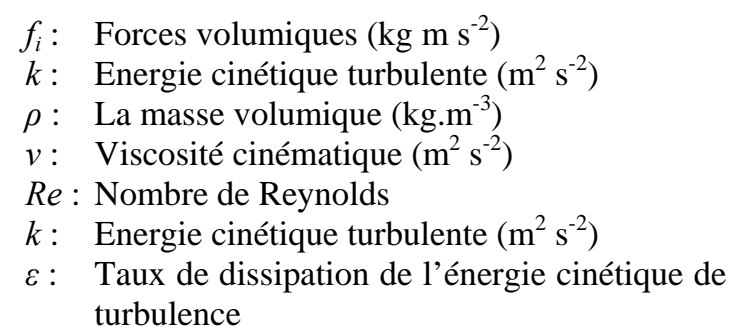

\section{Références bibliographiques}

BELCAID A., LEPALEC G., DRAOUI A., BOURNOT P. (2011). La modélisation de la diffusion de polluants dans un milieu marin. Application à la pollution de la baie de

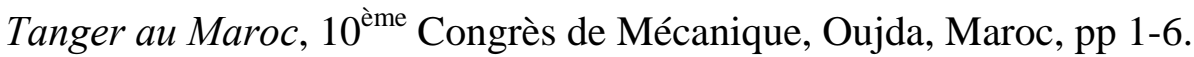

COUSTEIX J. (1989). Turbulence et couche limite. Cepadues Editions, Toulouse.

DUMAS R., FULACHIER L., ARZOUMANIAN E., FAVRE A. (1976). Étude de la structure de la turbulence dans une couche limite par les corrélations spatiotemporelles. J. Phys. Colloques 37, pp C1-181 - C1-184. doi:10.1051/jphyscol:1976127 FLUENT INC. (2005). Fluent 6.2 User's Guide. January 2005.

EL HATIMI I., EL ACHAB M., EL MOUMNI B. (2002). Impact des émissaires et canalisation sur l'environnement de la baie de Tanger (Maroc), approche géochimique. Bulletin de l’Institut Scientifique, Rabat, Section Sciences de la Terre, n²4, pp 49-58. PROJET SMAP III (2007). Sensibilisation et création d'un cadre politique favorisant l'intégration de l'environnement et du développement avec l'accent sur la région intégrée des zones côtières. Pollution côtière et développement durable, Rabat, Maroc. 